\title{
ILEAL ATRESIA CONCURRENT WITH AGENESIS OF THE ILEOCAECAL VALVE AND INTESTINAL VOLVULUS IN A GOAT KID
}

\author{
R. KHEIRANDISH ${ }^{1} \&$ J. TAJIK ${ }^{2}$ \\ ${ }^{1}$ Department of Pathobiology, ${ }^{2}$ Department of Clinical Sciences; School of \\ Veterinary Medicine, Shahid Bahonar University of Kerman, Kerman, Iran
}

\section{Summary}

Kheirandish, R. \& J. Tajik, 2017. Ileal atresia concurrent with agenesis of the ileocaecal valve and intestinal volvulus in a goat kid. Bulg. J. Vet. Med., 20, No 1, 73-79.

\begin{abstract}
Atresia of the distal ileum concurrent with ileocaecal valve agenesis is one of the rare types of intestinal atresia with very few reports in the literature. We herein present a case of type II distal ileal atresia, ileocaecal valve agenesis and volvulus of the ileum and the terminal jejunum with a history of depression, anorexia and failure to pass meconium. On necropsy, volvulus of the ileum and the terminal jejunum was found. The ileum was distended, full of fluid and gas, and similar to a blind-end sac. The ileocaecal valve was absent and the ileum was attached to the caecum by a fibrous band. The caecum was atrophied and small. The distal segments of the intestine were smaller than normal. These findings revealed type II terminal ileal atresia simultaneously agenesis of the ileocaecal valve and intestinal volvulus probably secondary to atresia in a newly born goat kid.
\end{abstract}

Key words: goat, ileocaecal atresia, ileocaecal valve agenesis

Intestinal atresia is a significant and lifethreatening problem leading to some complications including bowel obstruction and gastrointestinal disturbances (Dawrant et al., 2008). Congenital atresia of the intestinal tract is described as the complete closure of the intestinal lumen (Gelberg, 2012). It has been reported in human beings and many species of domestic mammals such as piglets, lambs, calves, pups and kittens (van der Gaag \& Tibboel, 1980; Kilic \& Sarierler, 2004; Radostitis et al., 2007; Azizi et al., 2010). It is likely to involve any intestinal segment from the duodenum to the anus undergo atresia (Kilic \& Sarierler, 2004; Azizi et al., 2010). In animals, atresia of the small intestine is less common than that of the large intestine (Brown et al., 2007; Radostitis et al., 2007). Although ileal atresia is the most common in calves, it is rare in other mammals such as lambs and kids (Brown et al., 2007). In calves, the most common sites have been reported to be the colon and anus followed by the jejunum and the ileum (Kilic \& Sarierler, 
2004; Brown et al., 2007; Lejeuner et al., 2011). The affected newborns may die due to autointoxication within a few days after birth (Radostitis et al., 2007). Intestinal volvulus is another life-threating condition in which a segment of the intestine twists on the mesenteric axis ( $\mathrm{Yu}$ et al., 2013). It may cause complications or lesions including vascular obstruction, congestion, haemorrhage, ischaemic injury and infarction (Gelberg, 2012). Intestinal atresia in approximately $25 \%$ of cases is followed by intestinal volvulus (Ogunyemi, 2000; Yu et al., 2013). Here, we present congenital type II terminal ileal atresia with the absence of the ileocaecal valve and volvulus of the ileum and the terminal part of the jejunum in a newborn goat.

A 3-day-old female domestic goat kid was presented with a history of depression, abdominal pain, bruxism, and anorexia. Meconium excretion had not been observed in the first 12 hours after birth and colostrum ingestion. However, there was no problem with the anal opening.
The owner had not performed any therapeutic approach. Anamnestic data did not reveal any previous cases of anomaly in the herd. Clinical examination revealed normal temperature, increased heart and respiratory rate, hyperaemic mucous membranes and slight abdominal distension. Based on the history and clinical evidence, we made a provisional diagnosis of intestinal atresia. Due to no opportune treatment, serious physical condition of the animal and the poor prognosis associated with surgical correction, the owner refused further diagnostics and treatment, and opted humane euthanasia. At necropsy, the duodenum and the early and intermediate portions of the jejunum were normal. Despite this, the terminal portion of the jejunum and the ileum were dark red and affected with volvulus and resultant necrosis and congestion (Fig. 1). Additionally, the ileum was found as a blind-end, enlarged and distended sac by the feces, air and fluid because of distal obstruction (type II or cord atresia) (Fig. 2). Meconium was found even in the distal

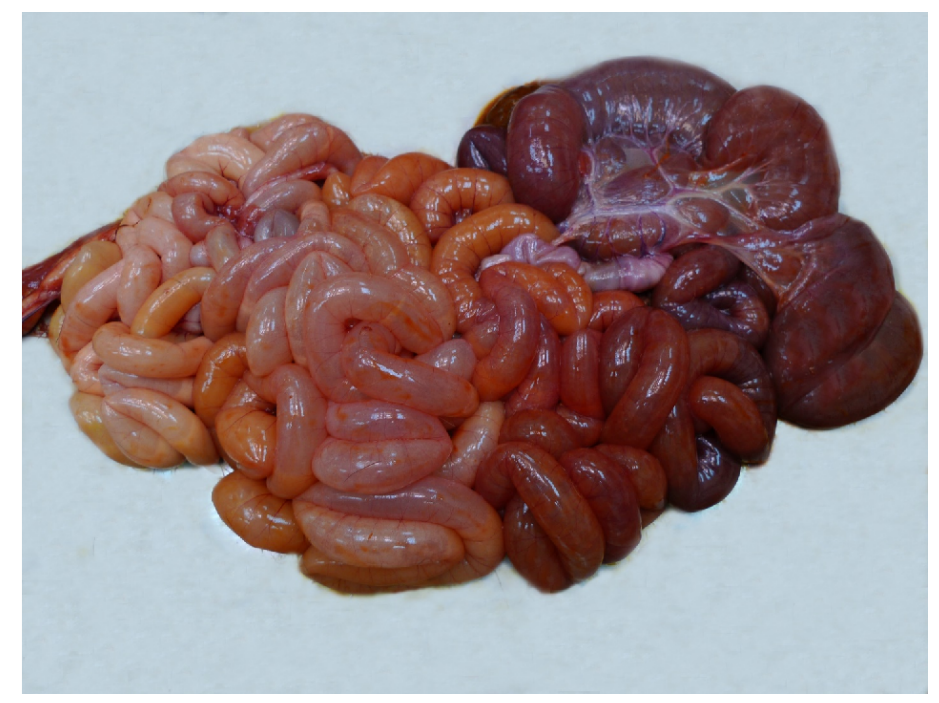

Fig. 1. Photograph shows volvulus of small intestine as dark red portion. 


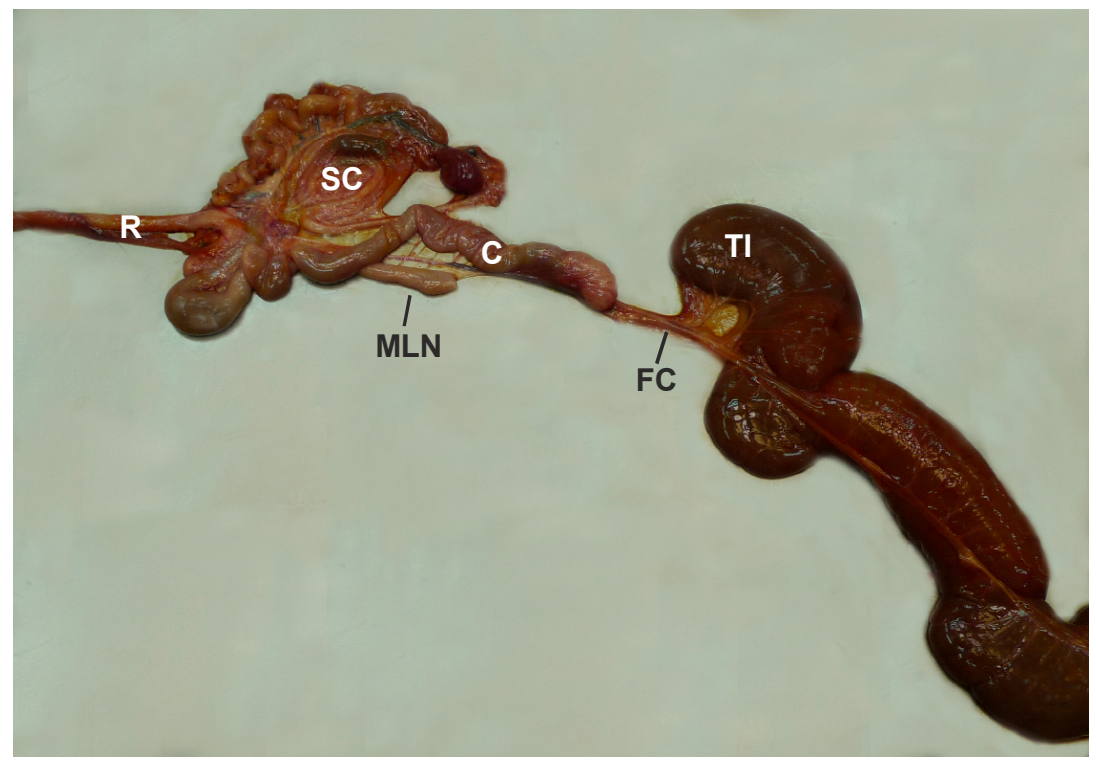

Fig. 2. Type II or cord atresia. Terminal ileum (TI) is seen as a blind-end sac distended by the faeces, joined to the caecum (C) by a fibrous cord (FC). Spiral colon (SC) and rectum are atrophied. Mesenteric lymph nodes (MLN) are normal.

segments to the atresia site. Moreover, the ileocaecal valve and orifice were absent and the ileum was joined to the caecum by a band of fibrous tissue. The caecum was small and atrophic and appeared as a narrow tube-like structure. The overall colon was atrophic, smaller and shorter than normal. In addition, the mesentery with its blood vessels supplying the ileocaecal region was absent. The mesenteric lymph nodes were normal and there was no evidence of any lesions. The findings obtained from necropsy revealed diagnosis of atresia of the terminal ileus (type II) with ileocaecal valve agenesis. No other anomalies were found in other organs.

The history and clinical signs of abdominal distention, anorexia, depression, lack of faeces in the rectum, and failure of passing meconium are all an indication of intestinal atresia (Elsa \& Onyeyili, 2004; Radostitis et al., 2007; Abouelnasr et al., 2012). However, observation of these signs in calves less than eight days old suggests intestinal atresia (Abouelnasr et al., 2012). Although intestinal atresia is a relatively common malformation, those that involve the ileocaecal region including ileocaecal valve and the associated mesentery with its blood vessels are considered to be extremely rare and little is known regarding their origin (Husaric et $a l ., 2012$ ). Intestinal atresia has been classified into several types (Kilic \& Sarierler, 2004; Gelberg 2012). Type I or membrane atresia is caused by a complete diaphragm or membrane. In type II named cord atresia, blind ends join via a small cord of fibrous or muscular tissue, and with or without mesentery. Type III or blind-end atresia is characterised by absence of a segment of the intestine, with disconnected blind ends and a gap in the mesentery, and often a short small intestine. Finally, in type IV, atresia involves multiple intestinal segments (Kilic \& Sari- 
erler, 2004). Moreover, there is a further type called apple peel or Christmas tree type that is a variant of type III atresia. In this type, the jejunum ends blindly and the blind end of the ileum is wrapped around the ileocolic artery (Kilic \& Sarierler, 2004; Gelberg, 2012). Therefore, the connecting fibrous band in the present case would classify it as type II atresia of the ileocaecal region with complete absence of the ileocaecal valve and the mesenteric defect. Intestinal atresia has been classified into several types that in type II named cord atresia, blind ends join via a small cord of fibrous or muscular tissue, with or without mesentery and lack a lumen (Kilic \& Sarierler, 2004). In the veterinary literature, some studies have been carried out in the field of intestinal atresia. In one study, the authors found 34 cases of atresia $(n=29)$ or stenosis $(n=5)$ of the small intestine in animals. There were 27 in the calves, five in the lambs, and two in the pups. Of 29 cases of intestinal atresia, the most common types were III (19), II (six), and I (four), respectively. In this study, most intestinal atresia cases was found in calves (van der Gaag \& Tibboel, 1980). In another study by Azizi et al. (2010), 68 of 492 newborn calves had intestinal atresia. In their study, no sex predilection was identified. In another investigation on 34 calves with intestinal atresia, 18, 13 and 3 cases had atresia of anus, anus and rectum and anus, rectum and distal colon, respectively (Durmus, 2009). Considering these studies, it can concluded that atresia of the ileum rarely occurs in animals except calves.

In spite of the literature concerning colonic atresia suggesting a non-heritable trait, the etiopathogenesis of ileal atresia remains incompletely understood (Elsa \& Onyeyili, 2004). However, there are two theories in this regard: one theory says that atresia arises from intra-uterine vascular insufficiency probably due to intussusception, volvulus, herniation or strangulation of the intestines during pregnancy period (Dawrant et al., 2008; Lejeuner et al., 2011; Gelberg, 2012). Moreover, the occlusion of the mesenteric vessels has been hypothesised as the etiology of ileal atresia in the medical literature (Husaric et al., 2012). Another theory suggests that atresia results from failure of revascularisation or recanalisation of the intestinal development. Although atresia of the duodenum is thought to be failure in embryonic recanalisation of lumen, most cases of non-duodenal atresia are believed to result from vascular impairment (Cho et $a l ., 2004)$. The event resulting in ischemic necrosis of a segment of the intestine may develop after the onset of fetal swallowing, so that meconium may be present in the distal segment to the atresia (Cho et al., 2004). In one study by Khen-Dunlop et al. (2009), jejunoileal atresia was experimentally created in rat by intestinal ligation and not by focal mesenteric ischemia. Nevertheless, doubt about the veracity of latter theory has led to more accuracy of the first one. Another rare form of intestinal atresia with a genetic cause has also been described (Dawrant $e t$ $a l ., 2008)$. In terms of genetic factors, atresia of the ileum in Swedish Highland cattle is inherited as autosomal recessive traits after inbreeding with a same bull (van der Gaag \& Tibboel, 1980; Lejeuner et al., 2011). Dawrant et al. (2008) demonstrated that injection of adriamycin leads to multiple malformations including multiple gastrointestinal atresia in an experimental study. Congenital anomalies mostly occur because of either genetic or environmental factors, or a combination of both (Kumar et al., 2009). There was no history regarding our case's dam ha- 
ving taken any medication during pregnancy. Furthermore, since no case with such anomalies had previously been observed on the farm, the probability of the genetic cause was rejected. It seems that ischaemia of the ileocaecal region is the most likely theory for explaining the pathogenesis of intestinal atresia in the present case as it was not associated with other congenital anomalies. Meanwhile, because the current case was associated with the presence of meconium and colostrum in the distended segment and meconium alone in those located after the atretic region, we postulate that the discussed atresia can be a late event during pregnancy. In our case, an additional finding was volvulus with necrosis and congestion in the ileum and the distal portion of the jejunum, proximal to the atretic region. It is unclear whether volvulus is secondary to ileal atresia or vice versa. In fact, intestinal atresia can develop after or before the volvulus. On one hand, as stated above and consistent with $\mathrm{Yu}$ et al. (2013), atresia can result from intrauterine volvulus causing ischaemia followed by atresia of the associated intestine. On the other hand, Ogunyemi (2000) showed that ileal atresia could possibly occur first and therefore, increased peristalsis in the dilated intestine before the atresia could result in volvulus. Regarding the discussed ileal atresia case, the authors believe that it has led to volvulus causing obstruction of the intestine, distention by gas or fluid and impairing intestinal peristalsis before atresia. Meanwhile, the existence of both meconium and colostrum and no meconium alone before and after the part with volvulus can support this supposition. Indeed, colostrum existence after volvulus confirms that volvulus has been caused after atresia that colostrum has passed from the region of volvulus but no atresia. Among 29 cases of intestinal atresia, Van der Gaad \& Tibboel (1980) reported a lamb with type III jejunal atresia concurrent with torsion of the cranial part of the blind-end atretic jejunum. In addition to these anomalies, the case was accompanied by atrophy of the subsequent segments to the atretic portion that it has not been previously reported. Presumptive diagnosis of intestinal atresia and volvulus is based on case history and clinical signs such as anorexia, depression, and abdominal distension or absence of defecation in newborns aged one to six days (Kilic \& Sarierler, 2004). Definitive diagnosis particularly those in more proximal locations such as ileal atresia needs further diagnostic approaches like radiography, ultrasonography and exploratory laparotomy (Azizi et al., 2010). Early diagnosis, surgical correction and supportive treatment are critical in the field of management of ileal atresia and volvulus and can rapidly return such cases to a normal state and prevent prolonged morbidity (Azizi et al., 2010). Anatomical typing of atresia should be performed to help determining the type of surgical correction for each case (Azizi et al., 2010). The treatment is based on the resection of the atretic segment followed by intestinal anastomosis (Khen-Dunlop et al., 2009; Husaric et al., 2012). Elsa \& Onyeyili (2004) reported two cases of goat kids affected with jejunal atresia corrected successfully by reseating and anastomosing the blind end so that the lumen of the intestine was patent for normal physiological function. Contrary to this, Abouelnasr et al. (2012) have stated that in most animals with intestinal atresia like those of proximal sites, surgical treatment is not recommended due to limited accessibility, low chance of postoperative survival rate and economic considerations. Moreover, 
if the cause of atresia is genetic origin and/or the affected animal is intended for breeding, surgery is not recommended due to probable propagation of genetic defects (Abouelnasr et al., 2012). Nonetheless, it has been shown that when the ileocaecal valve is completely absent, ileocaecal anastomosis appears to be sufficient (Cserni et al., 2006).

In summary, atresia of the terminal ileum concurrent with ileocaecal valve agenesis, the mesenteric defect and volvulus proximal to the atretic portion that we presented here is extremely rare and yet the first and unique report in the veterinary literature. We hope this case will represent an addition to the current literature on this topic and also provide more information regarding pathogenesis of intestinal atresia to pathologists and surgeons. Intestinal atresia should be noticed for several days in animals and treated at early stages.

\section{REFERENCES}

Abouelnasr, K., M. Ishii, H. Inokuma, Y. Kobayashi, K. Lee \& K. Yamada, 2012. Atresia coli in a Japanese black calf diagnosed by a barium sulphate enema contrast radiograph in the standing position: A case report. Veterinary Medicine, 57, 376-379.

Azizi, S., R. Mohammadi \& I. Mohammadpour, 2010. Surgical repair and management of congenital intestinal atresia in 68 calves. Veterinary Surgery, 39, 115-120.

Brown, C. C., D. C. Baker \& I. K. Barker, 2007. Alimentary system. In: Pathology of Domestic Animals, $5^{\text {th }}$ edn, eds K. V. F. Jubb, P. C. Kennedy \& N. Palmer, Elsevier, Guelph, p. 85.

Cho, F. N., T. L. Yang, Y. Y. Kan \& P. K. Sung, 2004. Prenatal sonographic findings in a fetus with congenital isolated ileal atresia. Journal of the Chinese Medical Association, 67, 366-368.
Cserni, T., A. Magyar, T. Nemeth, T. S. Paran, I. Csizy \& T. Jozsa, 2006. Atresia of the ileocecal junction with agenesis of the ileocecal valve and vermiform appendix: Report of a case. Surgery Today, 36, 1126-1128.

Dawrant, M. J., S. Giles, J. Bannigan \& P. Puri, 2008. Adriamycin produces a reproducible teratogenic model of gastrointestinal atresia in the mouse. Pediatric Surgery International, 24, 731-735.

Durmus, A. S., 2009. Congenital intestinal atresia in calves. Indian Veterinary Journal, 86, 737-738.

Elsa, A. T. \& P. A. Onyeyili, 2004. Surgical management of small intestinal atresia in Sokoto red goats. Pakistan Journal of Biological Science, 7, 2024-2025.

Gelberg, H. B., 2012. Alimentary system. In: Pathologic Basis of Veterinary Disease, $5^{\text {th }}$ edn, eds J. M. Zachary \& M. D. McGavin, Mosby Elseviers, Missouri, p. 361, 366.

Husaric, E., N. Hotic, A. Halilbasic, E. Konjic \& Z. Karasalihovic, 2012. Ileocecal atresia secondary to intrauterine intussusception. Pediatric Today, 8, 147-150.

Khen-Dunlop, N., L. Fourcade, F. Sauvat, G. de Lambert, A. Victor, N. Cerf-Bensussan \& S. Sarnacki, 2009. Surgical experimental jejunoileal atresia in rat embryo. Journal of Pediatric Surgery, 44, 1725-1729.

Kilic, N. \& M. Sarierler, 2004. Congenital intestinal atresia in calves: 61 cases (19992003). Revue de Médecine Vétérinaire, 155, 381-384.

Kumar, H., A. K. Sharma, L. L. Dass \& A. Anand, 2009. Atresia ani with scrotal anomaly in a goat. Veterinary World, 2, 68.

Lejeuner, B., J. Miclard, M. H. Stoffel \& M. Meylan, 2011. Intestinal atresia and ectopia in a bovine fetus. Veterinary Pathology, 48, 830-833.

Ogunyemi, D., 2000. Prenatal ultrasonographic diagnosis of ileal atresia and volvulus 
in a twin pregnancy. Journal of Ultrasound in Medicine, 19, 723-726.

Radostitis, O. M., C. C. Gay, K. W. Hinchcliff \& P. D. Constable, 2007. Veterinary Medicine: A Textbook of the Diseases of Cattle, Sheep, Pigs, Goats and Horses, $10^{\text {th }}$ edn, Saunders, Philadelphia, p. 280, 281.

Van der Gaag, I. \& D. Tibboel, 1980. Intestinal atresia and stenosis in animals: A report of 34 cases. Veterinary Pathology, 17, 565-574.

Yu, W., C. Ailu \& W. Bing, 2013. Sonographic diagnosis of fetal intestinal volvulus with ileal atresia: A case report. Journal of Clinical Ultrasound, 41, 255-257.
Paper received 24.07.2015; accepted for publication 12.11.2015

\section{Correspondence:}

Reza Kheirandish

Department of Pathobiology,

School of Veterinary Medicine,

Shahid Bahonar University of Kerman,

Kerman, Iran,

e-mail: kheirandish@uk.ac.ir 